\title{
The backward Îto method for the Lagrangian simulation of transport processes with large space variations of the diffusivity
}

\author{
D. Spivakovskaya ${ }^{1}$, A. W. Heemink ${ }^{1}$, and E. Deleersnijder ${ }^{2}$ \\ ${ }^{1}$ Department of Mathematical Physics, Delft Institute of Applied Mathematics (DIAM), Delft University of Technology, \\ Mekelweg 4, 2628 CD Delft, The Netherlands \\ ${ }^{2}$ Université catholique de Louvain, G. Lemaitre Institute of Astronomy and Geophysics (ASTR) \& Centre for Systems \\ Engineering and Applied Mechanics (CESAME), Avenue G. Lemaitre 4, 1348 Louvain-la-Neuve, Belgium
}

Received: 5 June 200 - Published in Ocean Sci. Discuss.: 17 July 2007

Revised: 13 November 2007 - Accepted: 2 December 2007 - Published: 20 December 2007

\begin{abstract}
Random walk models are a powerful tool for the investigation of transport processes in turbulent flows. However, standard random walk methods are applicable only when the flow velocities and diffusivity are sufficiently smooth functions. In practice there are some regions where the rapid but continuous change in diffusivity may be represented by a discontinuity. The random walk model based on backward Itto calculus can be used for these problems. This model was proposed by LaBolle et al. (2000). The latter is best suited to the problems under consideration. It is then applied to two test cases with discontinuous diffusivity, highlighting the advantages of this method.
\end{abstract}

\section{Introduction}

The transport of a tracer can be described by using the advection-diffusion equation. In general, this equation cannot be solved analytically, so that numerical methods must be resorted to. The most popular method is an Eulerian approach, in which the transport equation is solved on a fixed spatial grid. The finite element method and finite difference method are primary examples of this class of solution methods.

An alternative method is the Lagrangian approach, which follows particles through space at every time step. The movement of an individual particle is usually modeled in two steps: the advection, which is deterministic, is simulated by a translation of each particle with a velocity derived from the local fluid velocity field. Diffusion is generally simulated using stochastic methods. Then, by averaging the positions of many particles the advection-diffusion processes can be described (Thomson, 1987; Sawford, 1993; Costa and Ferreira,

Correspondence to: D. Spivakovskaya

(d.spivakovskaya@ewi.tudelft.nl)
2000; Zimmermann et al., 2001; Proehl et al., 2005; Delhez and Deleersnijder, 2006).

Particle-tracking models offer advantages over Eulerian methods in several respects. First, the solution obtained by using the particle tracking method is always mass conservative and non-negative, while the Eulerian methods is susceptible to excessive numerical dispersion and artificial oscillations (Zheng and Bennett, 2002) for advective dominated problems or problems with large gradients on the initial concentration field. Second, for problems where the tracer does not occupy the whole model domain, the Lagrangian methods models may be computationally more efficient than their Eulerian counterparts (Hunter, 1987; Spivakovskaya et al., 2005). Third, if the velocity field can be locally described by an analytic function, then particles may be advected exactly through that field by simple integration (Hunter et al., 1993). However, it should be noted, that the numerical flow can affect the accuracy of the particle tracking method. In this case the interpolation of flow variables in arbitrary particle location that can lead to local mass balance error and solution anomalies (LaBolle et al., 1996).

In general, both approaches have their own advantages and disadvantages: for instance, the Lagrangian approach can be an alternative to the Eulerian methods in case of steep concentration profile. On the other hand, the Eulerian approach is more suitable for dispersion-dominated problems, for which it provides accurate solution in reasonable time. The choice of a method depends on the problem under consideration. Sometimes, it is not easy to classify the problem and decide which method should be applied. The mixed Eulerian-Lagrangian methods attempt to combine the advantages of Lagrangian and Eulerian methods (Konikow and Bredehoeft, 1978; Celia et al., 1990; Yeh, 1990; Zhang et al., 1993; Zheng and Wang, 1999).

For space-varying diffusivity the advection part of the random walk model requires an additional correction term, which is proportional to the diffusivity gradient. Because of

Published by Copernicus Publications on behalf of the European Geosciences Union. 
this correction term the particles do not accumulate in regions of low diffusivity (Hunter et al., 1993; Visser, 1997; Ross and Sharples , 2004). This random walk model can be introduced by using the theory of stochastic differential equations (SDE) (Heemink, 1990; Dimou and Adams, 1993; Stijnen et al., 2006; Spivakovskaya et al., 2007). The advectiondiffusion equation is interpreted as the Fokker-Planck equation (Oksendal, 1985) and the corresponding SDE in Îto sense can be derived. As a result, the particle's track is simulated by a stochastic process, whose transition density function coincides with the tracer concentration. The Îto formulation is not the only way to introduce the particle tracking model. Another random walk model based on Stratonovich stochastic calculus is also quite popular.

Unfortunately, the common random-walk methods for simulating transport can only be applied when the diffusivity is sufficiently smooth, otherwise the correction term in the advection part dominates the flow velocity. In many situations the rapid but continuous change in turbulence statistics that occurs may be represented by a discontinuity (Thomson et al., 1997). Even without the large gradients, numerical simulation of the flow can result in discontinuities in the velocity field and, therefore, the velocity-dependent dispersion tensor may become discontinuous. One of the method to treat this problem is to interpolate the velocities in order to generate a smooth dispersion field (LaBolle et al., 1996). Recently LaBolle et al. (2000) proposed a random walk model based on backward Îto calculus that requires no corrective velocity.

In this paper, we discuss the random walk models based on Îto, Stratonovich and backward Îto calculus. The backward Itto random walk model is seen to be appropriate for dealing with a discontinuity in the diffusivity field. It is applied to two test cases, for which key properties of the solutions can be derived analytically.

\section{The Îto, Stratonovich and the backward Îto random walk models}

Let us consider the following one-dimensional advectiondiffusion problem:

$$
\frac{\partial C}{\partial t}=-\frac{\partial}{\partial x}\left(u C-k \frac{\partial C}{\partial x}\right)
$$

Here $C(t, x)$ is the concentration of a passive tracer, $u$ is flow velocity and $k(x)$ is diffusivity term. Equation (1) can be interpreted as a Fokker-Planck equation (see Karatzas and Shreve, 1998; Oksendal, 1985) and the corresponding Stochastic Differential Equation (SDE) in Îto sense can be considered

$$
\text { (I) } d X(t)=\left(u+k^{\prime}(x)\right) d t+\sqrt{2 k} d W(t)
$$

where $W$ is a Wiener process, i.e. a stochastic process with the following statistics $\left(t_{1} \leq t_{2} \leq t_{3} \leq t_{4}\right)$

$$
\begin{aligned}
& E\left(W\left(t_{2}\right)-W\left(t_{1}\right)\right)=0 \\
& E\left(\left(W\left(t_{4}\right)-W\left(t_{3}\right)\left(W\left(t_{2}\right)-W\left(t_{1}\right)\right)=0\right.\right. \\
& E\left(\left(W\left(t_{2}\right)-W\left(t_{1}\right)\right)\left(W\left(t_{2}\right)-W\left(t_{1}\right)\right)=t_{2}-t_{1}\right.
\end{aligned}
$$

Here $E(X)$ denotes the expectation of the random variable $X$. The solution of the advection-diffusion problem (1) is then th e probability density function of the stochastic process $X(t)$. The SDE (2) actually is not a "differential" equation, but can be interpreted as an integral equation

(I) $X(t)=x_{0}+\int_{0}^{t}\left(u+k^{\prime}(x)\right) d t+\int_{0}^{t} \sqrt{2 k} d W(t)$

The first integral (advection) in the right hand side of (4) is a standard Lebesgue integral, while the second part (diffusion) of (4) may be introduced as the limit of the sum (LaBolle et al. (2000))

(I)

$$
\begin{aligned}
& \int_{0}^{t} f(X, t) d W(t)= \\
& \text { ms- } \lim _{n \rightarrow \infty} \sum_{k=1}^{n} f\left(X\left(t_{k-1}\right), t_{k-1}\right)\left[W\left(t_{k}\right)-W\left(t_{k-1}\right)\right]
\end{aligned}
$$

Here $0=t_{0}<t_{1}<\ldots t_{n-1}=t_{n}=t$ and $\mathrm{ms}-\lim$ denotes the limit in the mean square sense. In general, to define unique stochastic integral one needs to specify at which point the function $f(X, t)$ is evaluated. For instance, in the definition of the Itto integral the function $f$ is always evaluated in the beginning of subinterval $\left[t_{k-1}, t_{k}\right]$ rendering $f\left(X\left(t_{k-1}\right), t_{k-1}\right)$ statistically independent of $\left[W\left(t_{k}\right)-W\left(t_{k-1}\right)\right]$ and thus ensuring that the Ito integral has zero mean. One well-known alternative, the Stratonovich integral, may be defined as a limit of the sum in which the function is evaluated at the middle of the time interval

$$
\begin{aligned}
& \int_{0}^{t} f(X, t) d W(t)= \\
& \text { ms- } \lim _{n \rightarrow \infty} \sum_{k=1}^{n} f\left(X\left(\frac{t_{k-1}+t_{k}}{2}\right), \frac{t_{k-1}+t_{k}}{2}\right) \times \\
& {\left[W\left(t_{k}\right)-W\left(t_{k-1}\right)\right]}
\end{aligned}
$$

The corresponding random walk model can be written as follows:

(S) $d X(t)=\left(u+\frac{1}{2} k^{\prime}(x)\right) d t+\sqrt{2 k} d W(t)$

The random walk models in the Îto or Stratonovich sense contain the diffusivity gradient in the advection part. For problems with large space variations of the diffusivity, this gradient may be very high and, therefore, dominates in the 
advection term. As a result, the solution obtained by the random walk model in the Îto or Stratonovich sense will not be accurate. To circumvent this diffusivity, one may have recourse to a random walk model that does not require a diffusivity gradient in the advection part. This formulation is based on the backward Itto integral (see Karatzas and Shreve (1998); LaBolle et al. (2000)).

(bI)

$$
\begin{aligned}
& \int_{0}^{t} f(X, t) d W(t)= \\
& \text { ms- } \lim _{n \rightarrow \infty} \sum_{k=1}^{n} f\left(X\left(t_{k}\right), t_{k}\right)\left[W\left(t_{k}\right)-W\left(t_{k-1}\right)\right]
\end{aligned}
$$

Using the backward Itto SDE for modelling advectiondiffusion processes with discontinuous diffusivity was proposed by LaBolle et al. (2000). The corresponding random walk model may be written as follows:

(bI) $d X(t)=u d t+\sqrt{2 k} d W(t)$

The sensitivity of the limit of the integral sums to the choice of location at which the function is evaluated is a consequence of the unbounded variation of the Wiener process (Karatzas and Shreve, 1998). However, each of the random walk methods introduced above is consistent with the advection-diffusion Eq. (1). For the continuous diffusion term, all these methods provide the same solution of Eq. (1).

Remark. The stochastic differential equations in the Îto, Stratanovich and backward Îto formulations are equivalent in case of smooth coefficients. When the coefficients are discontinuous the convergence of the stochastic differential equation in backward Ito formulation is not guaranteed. In LaBolle et al. (2000) the convergence of the backward Îto stochastic differential equations were proven for the one-dimensional case and demonstrated for two-dimensional case. However, there is no proof of the convergence for the multi-dimensional case. From the mathematical point of view, it would be more correct to use the term "generalized backward Îto" method to distinguish the difference between the stochastic differential equation with continuous and discontinuous parameters. For the sake of simplicity, we use the term "backward Îto" in this paper.

\section{Numerical integration of the SDEs}

It can be shown from the advection-diffusion Eq. (1) (see Hunter et al., 1993) that the mean and variance of the tracer cloud spread during time range $(t, t+\Delta t)$ are given by

$$
\begin{aligned}
& N_{1}=u^{i} \Delta t+k^{\prime}\left(X^{i}\right) \Delta t \\
& N_{2}-N_{1}^{2}=2 k\left(X^{i}\right) \Delta t+o(\Delta t) \approx 2 k\left(X^{i}\right) \Delta t
\end{aligned}
$$

Here $N_{i}, i=1,2$ denote the $i$ th moment of the concentration. Now we show that the first two moments of the displacement $\Delta X^{i}=X^{i+1}-X^{i}, i=1, \ldots, L$ in the random walk models (in
Îto, Stratonovich and backward Îto senses) are the same as the first moments of the concentration $C$.

Specific numerical schemes are associated with each of the stochastic methods mentioned above. For instance, the SDE in the Îto sense can be numerically integrated by applying the explicit Euler method:

$X^{i+1}=X^{i}+u^{i} \Delta t+k^{\prime}\left(X^{i}\right) \Delta t+\sqrt{2 k\left(X^{i}\right) \Delta t} R^{i}$

Here, $X^{i}=X\left(t_{i}\right), u^{i}=u\left(t^{i}, X^{i}\right), t_{i}=i \Delta t, i=0, \ldots, L-1$, $\Delta t=t / L$ and $R^{i}$ are mutually independent normally distributed random numbers with parameters $(0,1)$, e.g. the random variables with the following density function

$p(y)=\frac{1}{\sqrt{2 \pi}} e^{-\frac{y^{2}}{2}}$

We need only to find the probability law of the solution $X(t)$ of the SDE (in other words, solution in the weak sense), but not to approximate the solution itself. For these purposes, it is not necessary to chose normally distributed random variables. We can use any distribution with the same mean and variance, for instance, random numbers uniformly varying between $-\sqrt{3}$ and $\sqrt{3}$.

The solution obtained by using the random walk model (11) has the same properties

$$
\begin{aligned}
E\left(\Delta X^{i}\right)= & E\left(u^{i} \Delta t+k^{\prime}\left(X^{i}\right) \Delta t+\sqrt{2 k\left(X^{i}\right) \Delta t} R^{i}\right)= \\
& u^{i} \Delta t+k^{\prime}\left(X^{i}\right) \Delta t \\
\operatorname{Var}\left(\Delta X^{i}\right)= & E\left(\Delta X^{i}-\left(E\left(\Delta X^{i}\right)\right)\right)^{2}= \\
& E\left(\sqrt{2 k\left(X^{i}\right) \Delta t} R^{i}\right)^{2}=2 k\left(X^{i}\right) \Delta t
\end{aligned}
$$

As a result the solution obtained by this random walk model is consistent with the advection-diffusion Eq. (1).

The Heun method is more suitable for the Stratonovich formulation of the particle model (Kloeden and Platen, 1999)

$$
\begin{aligned}
& \Delta \tilde{X}^{i}=\sqrt{2 k\left(X^{i}\right) \Delta t} R^{i} \\
& X^{i}=X^{i-1}+u^{i} \Delta t+\frac{1}{2} k^{\prime}\left(X^{i}\right) \Delta t+ \\
& \frac{1}{2}\left(\sqrt{2 k\left(X^{i}\right) \Delta t}+\sqrt{2 k\left(X^{i}+\Delta \tilde{X}^{i}\right) \Delta t}\right) R^{i}
\end{aligned}
$$

Let us consider the mean and variance of $\Delta X^{i}$ obtained by method (14)

$$
\begin{aligned}
& E\left(\Delta X^{i}\right)=E\left(u^{i} \Delta t+\frac{1}{2} k^{\prime}\left(X^{i}\right) \Delta t\right. \\
& \left.+\frac{1}{2}\left(\sqrt{2 k\left(X^{i}\right) \Delta t}+\sqrt{2 k\left(X^{i}+\Delta \tilde{X}^{i}\right) \Delta t}\right) R^{i}\right)= \\
& u^{i} \Delta t+\frac{1}{2} k^{\prime}\left(X^{i}\right) \Delta t+\frac{1}{2} E\left(\sqrt{2 k\left(X^{i}+\Delta \tilde{X}^{i}\right) \Delta t} R^{i}\right)
\end{aligned}
$$




\begin{tabular}{|c|c|c|}
\hline \multicolumn{3}{|c|}{ Advection-diffusion equation } \\
\hline$\Downarrow$ & $\Downarrow$ & $\Downarrow$ \\
\hline Îto & Stratonovich & backward Îto \\
\hline$\Downarrow$ & $\Downarrow$ & $\Downarrow$ \\
\hline$t_{k}^{*}=t_{k-1}$ & $t_{k}^{*}=\left(t_{k}+t_{k-1}\right) / 2$ & $t_{k}^{*}=t_{k}$ \\
\hline$\Downarrow$ & $\Downarrow$ & $\Downarrow$ \\
\hline$d X=\left(u+\frac{\partial k}{\partial x}\right) d t+\sqrt{2 k} d W(t)$ & $d X=\left(u+\frac{1}{2} \frac{\partial k}{\partial x}\right) d t+\sqrt{2 k} d W(t)$ & $d X=u d t+\sqrt{2 k} d W(t)$ \\
\hline$\Downarrow$ & $\Downarrow$ & $\Downarrow$ \\
\hline Euler explicit & Heun & Euler backward \\
\hline$\Downarrow$ & $\Downarrow$ & $\Downarrow$ \\
\hline$\Delta X^{i}=\left(u^{i}+\left(k^{\prime}\left(X^{i}\right)\right) \Delta t+\sqrt{2 k\left(X^{i}\right) \Delta t} R^{i}\right.$ & $\begin{array}{l}\Delta \tilde{X}^{i}=\sqrt{2 k\left(X^{i}\right) \Delta t} R^{i} \\
\Delta X^{i}=\left(u^{i}+\frac{1}{2}\left(k^{\prime}\left(X^{i}\right)\right) \Delta t+\right. \\
\frac{1}{2}\left(\sqrt{2 k\left(X^{i}\right) \Delta t}+\sqrt{2 k\left(X^{i}+\Delta \tilde{X}^{i}\right) \Delta t}\right) R^{i}\end{array}$ & $\begin{aligned} \Delta \tilde{X}^{i} & =\sqrt{2 k\left(X^{i}\right) \Delta t} R^{i} \\
\Delta X^{i} & =u^{i} \Delta t+\sqrt{2 k\left(X^{i}+\Delta \tilde{X}^{i}\right) \Delta t} R^{i}\end{aligned}$ \\
\hline$\Downarrow$ & $\Downarrow$ & $\Downarrow$ \\
\hline \multicolumn{3}{|c|}{ Random walk model (consistent with the advection-diffusion equation) } \\
\hline
\end{tabular}

Fig. 1. Comparison between Îto, Stratonovich and backward Îto formulations.

Let us expand the function $B\left(X^{i}+\Delta \tilde{X}^{i}\right)=\sqrt{2 k\left(X^{i}+\Delta \tilde{X}^{i}\right)}$ into Taylor series

$$
\begin{aligned}
& B\left(X^{i}+\Delta \tilde{X}\right)=B\left(X^{i}\right)+B^{\prime}\left(X^{i}\right) \Delta \tilde{X}+O\left(\Delta \tilde{X}^{2}\right)= \\
& \sqrt{2 k\left(X^{i}\right)}+k^{\prime}\left(X^{i}\right) \sqrt{\Delta t} R^{i}+o(\Delta t) \approx \\
& \sqrt{2 k\left(X^{i}\right)}+k^{\prime}\left(X^{i}\right) \sqrt{\Delta t} R^{i}
\end{aligned}
$$

In other words the following equation is valid

$\sqrt{2 k\left(X^{i}+\Delta \tilde{X}^{i}\right)} \approx \sqrt{2 k\left(X^{i}\right)}+k^{\prime}\left(X^{i}\right) \sqrt{\Delta t} R^{i}$

Substituting (16) into (15) yields

$$
\begin{aligned}
& E\left(\Delta X^{i}\right)=u^{i} \Delta t+\frac{1}{2} k^{\prime}\left(X^{i}\right) \Delta t+\frac{1}{2} k^{\prime}\left(X^{i}\right) \Delta t E\left(R^{i}\right)^{2}= \\
& u^{i} \Delta t+k^{\prime}\left(X^{i}\right) \Delta t
\end{aligned}
$$

The variation of the displacement $\Delta X$ in the Heun scheme coincides with the variation of the concentration

$$
\begin{aligned}
& \operatorname{Var}\left(\Delta X^{i}\right)= \\
& E\left(\frac { 1 } { 2 } \left(\left(\sqrt{2 k\left(X^{i}\right) \Delta t}+\right.\right.\right. \\
& \left.\left.\left.\sqrt{2 k\left(X^{i}+\Delta \tilde{X}\right) \Delta t}\right) R^{i}-k^{\prime}\left(X^{i}\right) \Delta t\right)\right)^{2}= \\
& E\left(\sqrt{2 k\left(X^{i}\right) \Delta t} R^{i}+\frac{1}{2} k^{\prime}\left(X^{i}\right)\left(R^{i}-1\right) \Delta t\right)^{2}= \\
& 2 k\left(X^{i}\right) \Delta t+o(\Delta t) \approx 2 k\left(X^{i}\right) \Delta t
\end{aligned}
$$

We can conclude that the random walk model (14) has the same first two moments as a standard random walk model in the Îto sense and as in (10).

Finally, the backward Euler scheme is appropriate for the backward Itto formulation (see LaBolle et al., 2000)

$$
\begin{aligned}
& \Delta \tilde{X}^{i}=\sqrt{2 k\left(X^{i}\right) \Delta t} R^{i} \\
& X^{i}=X^{i-1}+u^{i} \Delta t+\sqrt{2 k\left(X^{i}+\Delta \tilde{X}^{i}\right) \Delta t} R^{i}
\end{aligned}
$$

Using Eq. (16) we can again find the moments of the distribution of $X(t)$ obtained by backward Euler scheme

$$
\begin{aligned}
& E\left(\Delta X^{i}\right)= \\
& E\left(u^{i} \Delta t+\sqrt{2 k\left(X^{i}+\Delta \tilde{X}^{i}\right) \Delta t} R^{i}\right)= \\
& u^{i} \Delta t+E\left(\left(\sqrt{2 k\left(X^{i}\right)}+k^{\prime}\left(X^{i}\right) \sqrt{\Delta t} R^{i}\right) \sqrt{\Delta t} R^{i}\right)= \\
& u^{i} \Delta t+k^{\prime}\left(X^{i}\right) \Delta t
\end{aligned}
$$

and

$$
\begin{aligned}
& \operatorname{Var}\left(\Delta X^{i}\right)= \\
& E\left(\sqrt{2 k\left(X^{i}+\Delta \tilde{X}^{i}\right) \Delta t} R^{i}-k^{\prime}\left(X^{i}\right) \Delta t\right)^{2}= \\
& E\left(\sqrt{2 k\left(X^{i}\right) \Delta t} R^{i}+k^{\prime}\left(X^{i}\right) \Delta t\left(R^{i}\right)^{2}-k^{\prime}\left(X^{i}\right) \Delta t\right)^{2}= \\
& 2 k\left(X^{i}\right) \Delta t+o(\Delta t) \approx 2 k\left(X^{i}\right) \Delta t .
\end{aligned}
$$

As a result the solution obtained by the backward Itto random walk model is consistent with the advection-diffusion Eq. (1). The main differences between the Ito, Stratonovich and the backward Ito formulation are shown in Fig. 1.

\section{Illustrations}

In this section the random walk models (in Itto and backward Ito senses) are applied to two test cases. In general, the analytical solution of the direct problem (1) cannot be found; however, the residence time of a tracer can be obtained (Delhez et al., 2004; Deleersnijder et al., 2006a,b). The residence time of a water or tracer parcel in a control domain is usually defined as the time taken by this parcel to leave the domain of interest (Bolin and Rodhe, 1973; Zimmerman, 1976, 


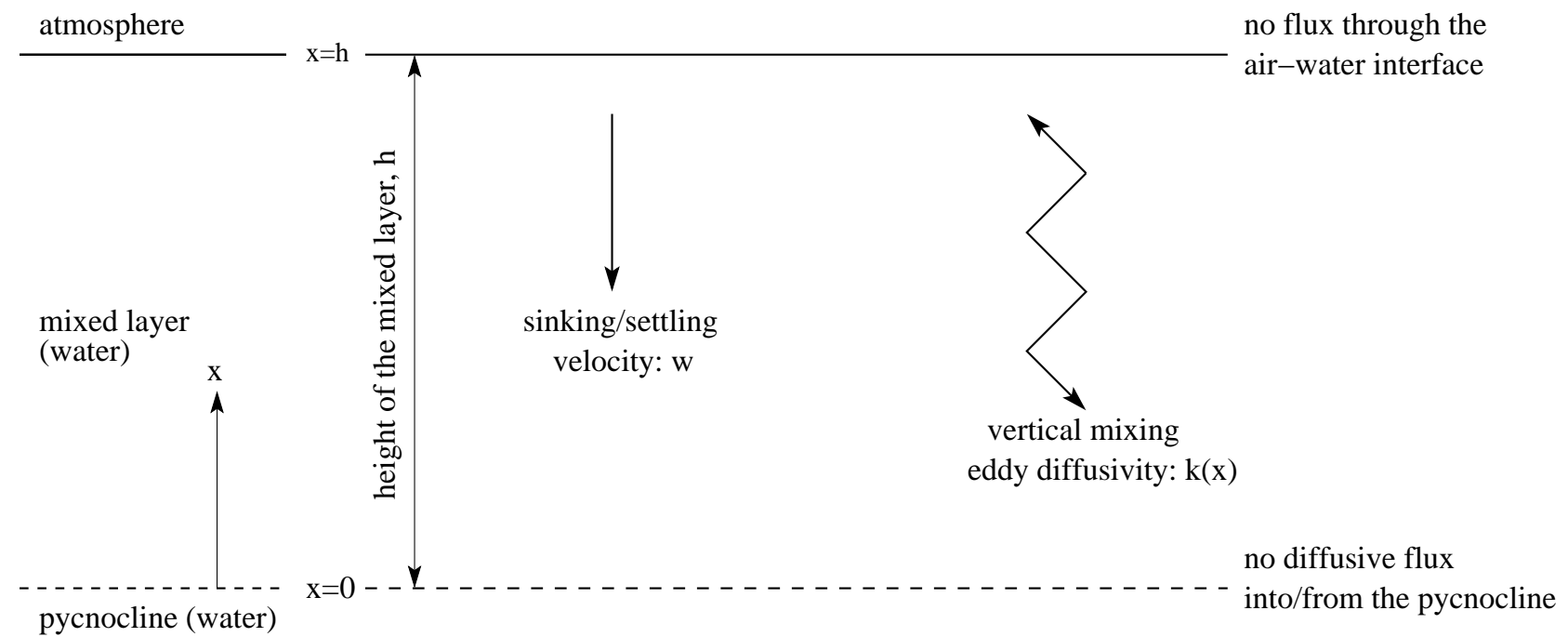

Fig. 2. Sinking-diffusion model: illustration of its geometry, parameters and boundary conditions. Source Deleersnijder et al. (2006a).

1988; Braunschweig et al., 2003; Takeoka, 1984; Delhez and Deleersnijder, 2006). As such, the residence time is one of the most popular tool to describe and understand environmental issues.

Mathematically, the mean residence time $\theta(x)$ of the tracer of initial mass $m\left(t_{0}\right)$ released at time $t_{0}$ can be computed by monitoring the temporal evolution of the mass of the tracer in the control region (Bolin and Rodhe (1973); Takeoka (1984))

$\theta(x)=-\frac{1}{m\left(t_{0}\right)} \int_{m\left(t_{0}\right)}^{0} t d m$

Delhez et al. (2004) introduced an alternative procedure designed for numerical models. They showed that the residence time can be found from the solution of the adjoint problem to the advection-diffusion equation.

For both examples, we assume that the diffusivity is discontinuous at some location. Such diffusivity profile does not exist in the nature; however, there are regions of large space variations of the diffusivity. The discontinuous diffusivity can be considered as a limit case for which it is generally easier to find the analytical solution. In addition, if the Lagrangian method under consideration can successfully handle a discontinuity in the diffusivity field, it is safe to assume that this method will be able to deal with region of high gradients of the eddy coefficient.

\subsection{Illustration 1: Settling and diffusion problem}

First, we apply the random walk model (9) to the settling and diffusion model (Fig. 2) proposed and analyzed by Deleersnijder et al. (2006a,b). In this model we assume that $x$ is a vertical coordinates that increases upwards. It is zero at the interface between the mixed layer and the underlying pycnocline. If $h$ is the height of the mixed layer, the water-air interface is located at $x=h . w$ represents the settling velocity (we assume that $w$ is a constant) and $k(x)$ is the vertical eddy diffusivity, which is positive in the interval $0<x<h$ and is zero in the pycnocline, i.e. underneath the domain of interest. We suppose that the upper boundary of the domain is impermeable

$\left[w C+k \frac{\partial C}{\partial x}\right]_{x=h}=0$

It is only by settling that the particles of the tracer under study can leave the domain of interest and enter the pycnocline, so the turbulent diffusion flux must be prescribed to be zero at the bottom of the mixed layer

$\left[k \frac{\partial C}{\partial x}\right]_{x=0}=0$

The initial concentration is

$C(0, x)=\delta\left(x-x_{0}\right)$

where $\delta(x)$ denotes Dirac delta function.

Deleersnijder et al. (2006b) showed that the residence time may exhibit a discontinuity at the interfaces between the mixed layer $(0<x<h)$ and pycnocline $(x<0)$, for the eddy diffusivity is zero in the latter and positive in the former. Now we assume that the boundary of interest is $x=\epsilon$, rather than $x=0 . \epsilon$ is positive or negative according to whether the boundary is located in the mixed layer or the pycnocline, respectively. The corresponding residence time is hereinafter denoted

$\theta\left(x_{0}, \epsilon\right)=\int_{0}^{\infty} \int_{\epsilon}^{h} C(t, x) d x d t$ 

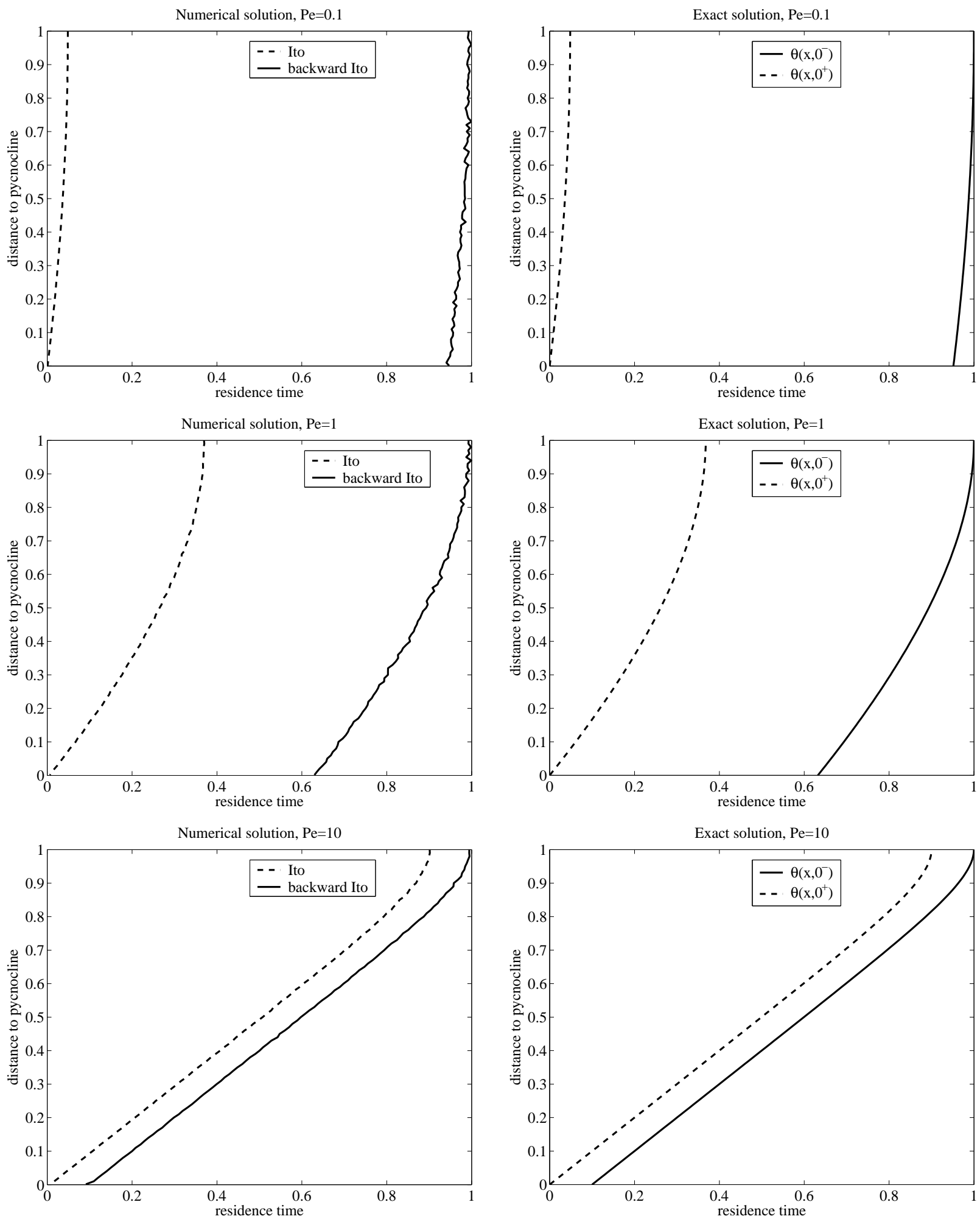

Fig. 3. The profile of the residence times $\theta\left(x, 0^{-}\right)$and $\theta\left(x, 0^{+}\right)$in the surface mixed layer for various values of the Peclet number. Dimensionless variables are used and the eddy diffusivity is assumed to be constant in the mixed layer. $\Delta t=10^{-4}, N=10^{5}$. 


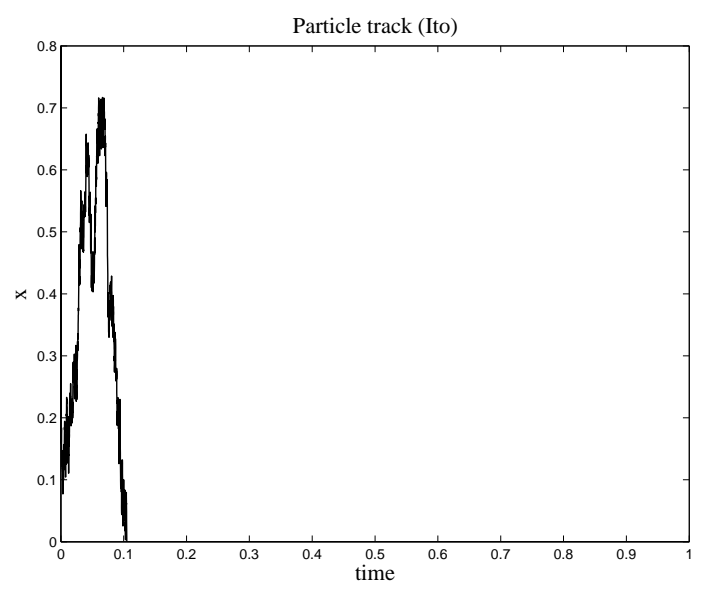

(a)

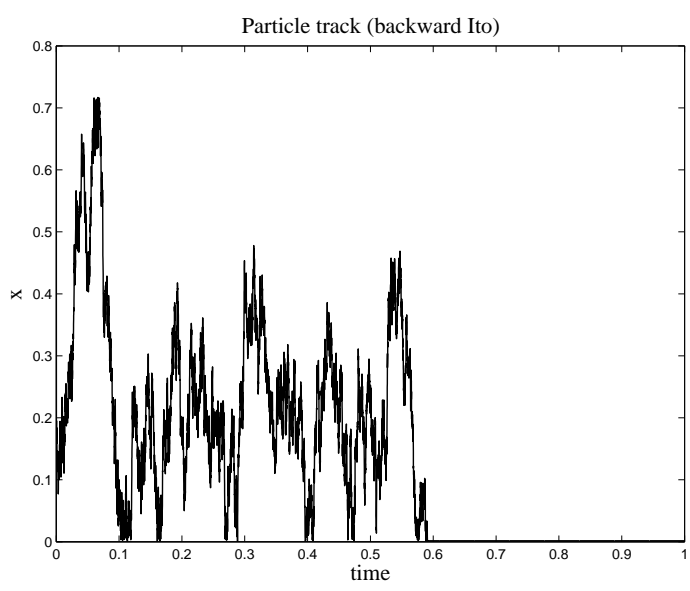

(b)

Fig. 4. The track of a particle released at location $x_{0}=0.1$ in case of (a) Îto and (b) backward Îto random walk models.

which may be recast as a function of $x$. There is no closed form solution for $C(t, x)$, but the residence time may be calculated analytically (Deleersnijder et al., 2006a).

For the sake of simplicity, it is assumed that the eddy diffusivity is a positive constant $\lambda$, in the mixed layer and zero in the pycnocline. It is also desirable to introduce dimensionless variables:

$x^{\prime}=\frac{x}{h}, \quad \theta^{\prime}=\frac{\theta}{h / w}, \quad P e=\frac{w h}{\lambda}$

From now on, only dimensionless variables will be used, so that it is appropriate to drop the primes. If $\epsilon>0$, the lower boundary is located at a level where the eddy diffusivity is nonzero, while $\epsilon<0$ corresponds to the case, when the lower boundary is located below the pycnocline. Let us assume that the lower boundary of the domain is pushed towards the bottom of the mixed layer $\epsilon>0, \epsilon \rightarrow 0^{+}, \epsilon<0, \epsilon \rightarrow 0^{-}$. Deleersnijder et al. (2006b) show that the corresponding residence times are different. In particular, for the chosen value of the diffusivity

$\theta\left(x, 0^{-}\right)=x+\frac{1-e^{-P e(1-x)}}{P e}$

and

$\theta\left(x, 0^{+}\right)=x-\frac{e^{-P e(1-x)}-e^{-P e}}{P e}$

We apply the Îto and the backward Îto random walk models to simulate the transport of the tracer in the proposed model. The Itto random walk model formulation corresponds to the case when the lower boundary of the domain is placed above the pycnocline, while the backward Îto random walk model provides the solution of the case when the lower boundary of the domain is placed under pycnocline.
The exact and the numerical solutions for $N=10^{4}$ particles are shown in Fig. 3.

From Fig. 3 we can conclude that the residence times obtained by applying the Îto and backward Îto random walk schemes are different. One can wonder which scheme provides the right solution. In reality, the both methods are correct, however they give answers for two different problems.

In Sect. 3 it was shown that for a smooth diffusivity function both random walk schemes are identical. In the Îto case, an additional drift due to the spatial variation of the diffusivity is present. Because of this additional drift particles cannot stay in regions with low diffusivity. In the backward Îto formulation the additional drift term has disappeared and is included in the random term by applying the two-steps backward Euler scheme.

The disadvantage of the Îto formulation is that it cannot handle the case of discontinuous diffusivity. By applying an Îto model in this case the diffusivity drift is zero everywhere except exactly at the boundary where it is infinite. By applying a numerical scheme, particles will never reach exactly the pycnocline and as a result the diffusivity drift becomes essentially zero. Therefore a particle that comes close to the boundary will never go back into the domain (see Fig. 4a) and the residence time computed is in fact the residence time $O\left(x, 0^{+}\right)$.

By applying the backward Îto model the diffusivity drift is included in the random term of the model. Now a particle does get back into the domain even if it is very close to the boundary (see Fig. 4b). So the presence of the pycnocline is taken into account, leading to the residence time $O\left(x, 0^{-}\right)$. 
4.2 Illustration 2: The direct and adjoint problems for the residence time

In the previous section we considered a model, in which the diffusivity exhibits a discontinuity at the boundary of the domain. However, in practice the diffusivity can change rapidly inside the domain of interest. An example of such a problem is needed. In this respect, inspiration may be found in Delhez and Deleersnijder (2006).

Let $t$ and $x$ denote time and a space coordinate, respectively. In the domain $-L \leq x \leq L$, the concentration of the tracer $C(t, x)$ obeys the following partial differential problem

$$
\begin{aligned}
& \frac{\partial C}{\partial t}=-\frac{\partial}{\partial x}\left(u C-k \frac{\partial C}{\partial x}\right), \\
& C\left(0, x, x_{0}\right)=\delta\left(x-x_{0}\right), \quad-L<x_{0}<L, \\
& C\left(t, \pm L, x_{0}\right)=0
\end{aligned}
$$

where the positive constant $u$ is the fluid velocity, while $k(x)>0$ denotes the eddy diffusivity. The residence time in the domain of interest of the tracer whose concentration obeys the partial differential problem (29) is (Delhez et al., 2004)

$$
\theta\left(x_{0}\right)=\int_{0}^{\infty} \int_{-L}^{L} C(t, x) d x d t
$$

In principle this value may be evaluated for any admissible value of $x_{0}$. The ensuing function may then be recast as a function of $x$, i.e. $\theta(x)$. However, obtaining the analytical solution of the direct problem (29) is usually considered as difficult. Fortunately, it is much easier to obtain the residence time by solving the adjoint problem (Delhez et al., 2004; Delhez and Deleersnijder, 2006):

$$
\begin{aligned}
& \frac{d}{d x}\left(k \frac{d \theta}{d x}+u \theta\right)=-1 \\
& \theta( \pm L)=0
\end{aligned}
$$

For the purposes of the present study, the eddy diffusivity must exhibit a discontinuity inside the domain of the interest. The simplest expression that satisfies this constraint probably is the following piecewise constant function

$k(x)=\left\{\begin{array}{l}k^{+}, 0<x \leq L \\ k^{-},-L \leq x<0\end{array}\right.$

where $k^{+}$and $k^{-}$are positive constants. Therefore, at $x=0$, the residence time must satisfy two matching conditions:

$[\theta(x)]_{x=0^{-}}^{x=0^{+}}=0$

$\left[k \frac{\partial \theta}{\partial x}+u \theta\right]_{x=0^{-}}^{x=0^{+}}=0$

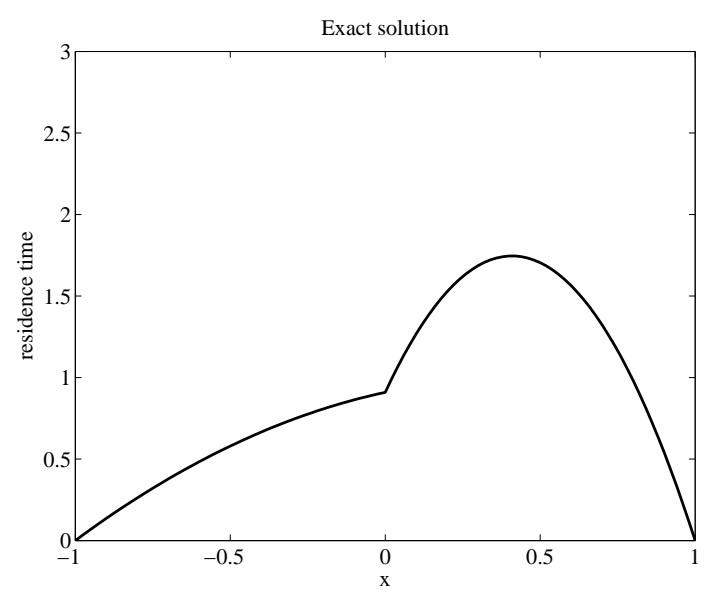

Fig. 5. The profile of the residence time in case of zero advection. Dimensionless variables were used. The value of parameter: $\mu=0.1$.

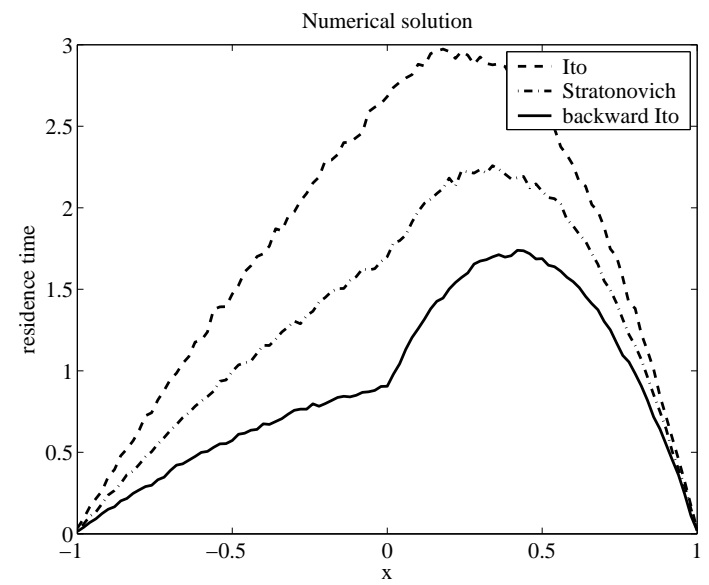

Fig. 6. The profile of the residence time in case of zero advection. Dimensionles s variables were used. Numerical solution for $N=10^{4}$ particles with time step $\Delta t=10^{-4}$.

In the developments below, the residence time at $x=0$ will be denoted $\theta_{0}$. In other words, the latter satisfies the equalities

$\theta\left(0^{-}\right)=\theta_{0}=\theta\left(0^{+}\right)$

\subsubsection{The zero advection case}

If the advection is zero $(u=0)$, then it is appropriate to introduce the dimensionless parameter $\mu=k^{+} / k^{-}$and variables

$$
\begin{aligned}
& t^{\prime}=\frac{t}{L /\left(k^{-}\right)^{2}}, \quad\left(x^{\prime}, x_{0}^{\prime}\right)=\frac{\left(x, x_{0}\right)}{L}, \quad k^{\prime}=\frac{k}{k^{-}}, \\
& C^{\prime}=\frac{C}{1 / L}, \quad\left(\theta^{\prime}, \theta_{0}^{\prime}\right)=\frac{\left(x, x_{0}\right)}{L /\left(k^{-}\right)^{2}}
\end{aligned}
$$


For the sake of simplicity the primes can be dropped. Hence, the dimensionless diffusivity is

$k(x)=\left\{\begin{array}{l}\mu, 0<x \leq 1 \\ 1,-1 \leq x<0\end{array}\right.$

After some calculations, the residence time is obtained:

$\theta(x)=\left\{\begin{array}{l}-\frac{x^{2}}{2 \mu}-\frac{2 \mu \theta_{0}-1}{2 \mu} x+\theta_{0}, 0<x \leq 1 \\ -\frac{x^{2}}{2}+\frac{2 \theta_{0}-1}{2} x+\theta_{0}, \quad-1 \leq x<0\end{array}\right.$

with

$\theta_{0}=\frac{1}{1+\mu}$

The analytical and numerical solutions obtained by Îto, Stratonovich and backward Ito random walk methods are shown on Figs. 5, 6 respectively. Clearly, the backward Ito solution is much better than the Stratonovich solution, which, in turn, is better than that obtained by the classical Îto method.

\subsubsection{The advection-diffusion case}

If advection is present $(u>0)$, then it is appropriate to introduce the following dimensionless parameters and variables:

$$
\begin{aligned}
& t^{\prime}=\frac{t}{L / u}, \quad\left(x^{\prime}, x_{0}^{\prime}\right)=\frac{\left(x, x_{0}\right)}{L}, \quad P e^{ \pm^{\prime}}=\frac{u L}{k^{ \pm}}, \\
& C^{\prime}=\frac{C}{1 / L}, \quad\left(\theta^{\prime}, \theta_{0}^{\prime}\right)=\frac{\left(x, x_{0}\right)}{L / u}
\end{aligned}
$$

As in the previous example the primes can be dropped. It is also useful to define a piecewise constant Peclet number:

$P e(x)=\left\{\begin{array}{l}P e^{+}, 0<x_{0} \leq 1 \\ P e^{-},-1 \leq x_{0}<0\end{array}\right.$

After some calculations, the residence time is obtained:

$\theta(x)=\left\{\begin{array}{l}a^{+}-x+b^{+} e^{-P e^{+} x}, 0<x \leq 1 \\ a^{-}-x+b^{-} e^{-P e^{-} x},-1 \leq x<0\end{array}\right.$

with

$a^{ \pm}=\frac{e^{\mp P e^{ \pm}} \theta_{0} \mp 1}{e^{\mp P e^{ \pm}}-1}, \quad a^{ \pm}=\frac{ \pm 1-\theta_{0}}{e^{\mp P e^{ \pm}}-1}$

and

$$
\begin{aligned}
& \theta_{0}=\frac{P e^{+}-P e^{-}}{P e^{+} P e^{-}} \frac{\left(e^{-P e^{+}}-1\right)\left(e^{-P e^{-}}-1\right)}{e^{-P e^{+}}-e^{-P e^{-}}}- \\
& \frac{e^{-P e^{+}}+e^{-P e^{-}-1}}{e^{-P e^{+}}-e^{-P e^{-}}}
\end{aligned}
$$

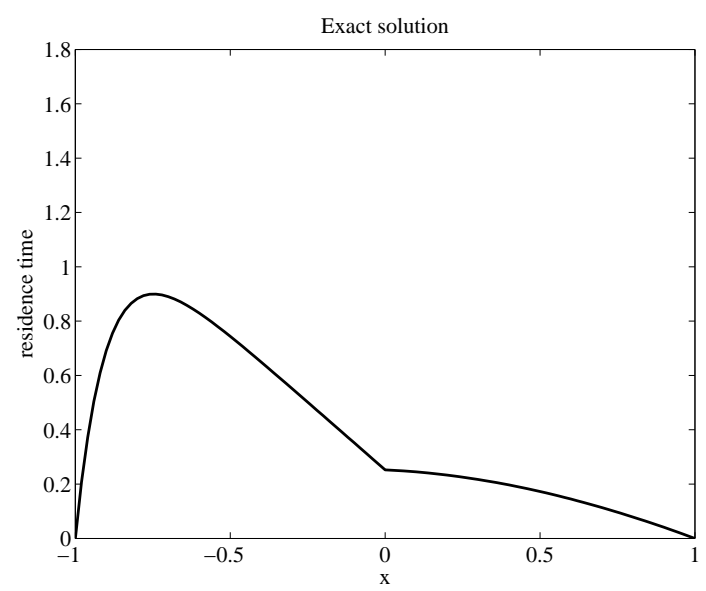

Fig. 7. The profile of the residence time in case of non zero advection. The value of parameter: $P e^{+}=0.5, P e^{-}=10$. Dimensionless variables were used.

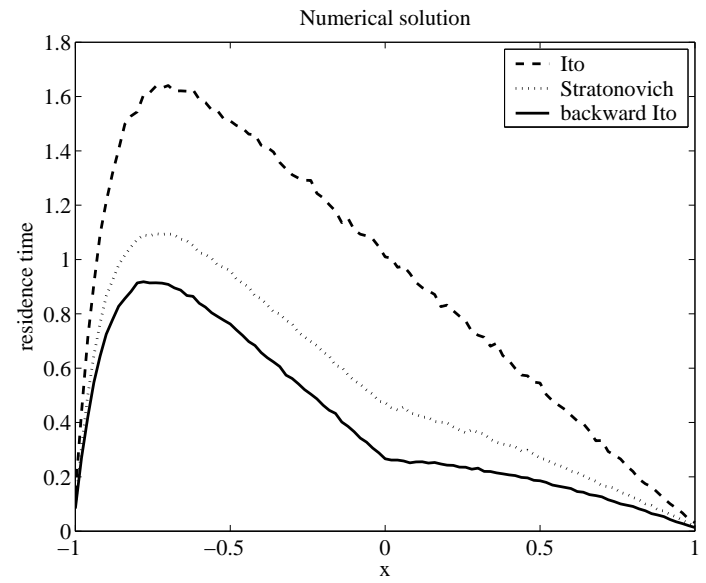

Fig. 8. The profile of the residence time in case of non zero advection. Numerical solution for $N=10^{4}$ particles, $P e^{+}=0.5, P e^{-}=10$. Dimensionless variables were used.

Figure 7 shows the analytical solution and the numerical solutions obtained from Îto, Stratonovich and backward Îto formulations are shown on Fig. 8. One can see easily that only the solution obtained by the backward Itto random walk model is very close to the analytical solution, while the Stratonovich and Îto solutions significantly differ from the exact residence time.

\section{Conclusions}

In this paper we considered the random walk model that can be applied to model the transport process in the regions with large space variations of the diffusivity, or as the limit 
case with discontinuous diffusivity. This model proposed by LaBolle et al. (2000) is based on the backward Îto stochastic integral. It is consistent with the advection-diffusion equation and does not contain the diffusivity gradient in the advection part. Two test cases were analyzed: a sinkingdiffusion model, in which the diffusivity exhibits a discontinuity at one boundary of the domain and an advectiondiffusion problem with a discontinuity in the diffusivity inside the domain of interest. For both test cases the analytical solution of the indirect problem, e.g. finding the residence time, is known. The backward Itto random walk model was applied and the results show that this model provides the correct results for discontinuous diffusivities, while other, better known, random walk models perform rather poorly.

Acknowledgements. Eric Deleersnijder is a Research Associate with the Belgian National Fund for Scientific Research (FNRS). His contribution to the present study was made in the framework of the Interuniversity Attraction Pole TIMOTHY (IAP. 13) which is funded by the Belgian Federal Science Policy Office (BELSPO).

Edited by: N. C. Wells

\section{References}

Bjøork, T.: Arbitrage Theory in Continuous Time, Oxford University Press, 1998.

Bolin, B. and Rodhe, H.: A note on concepts of age distribution and transit time in natural reservoirs, Tellus, 25, 58-62, 1973.

Braunschweig, F., Martins, F., Chambel, P., and Neves, R.: A methodology to estimate renewal time scales in estuaries: the Tagus Estuary case, Ocean Dynam., 53, 137-145,2003.

Celia, M. A., Russell, T. F., Herrera, I., and Ewing, R. E.: An Eulerian-Lagrangian localized adjoint method for an advectiondiffusion equation, Adv. Water Resour., 13(4), 187-206, 1990.

Costa, M. and Ferreira, J. S.: Discrete particle distribution model for advection-diffusion transport, J. Hydraul. Eng., 126(7), 525532, 2000

Deleersnijder E., Beckers J. M., and Delhez E. J. M.: The residence time of settling in the surface mixed layer, Environ. Fluid Mech., 6(1), 25-42, 2006a.

Deleersnijder E., Beckers J. M., and Delhez E. J. M.: On the behavior of the residence time at the bottom of the mixed layer, Environ. Fluid Mech., 6, 541-547, 2006b.

Delhez, E. J. M., Heemink, A. W., and Deleersnijder, E.: Residence time in a semi-enclosed domain from the solution of an adjoint problem, Estuarine, Coastal and Shelf Science, 61, 691702, 2004

Delhez, E. J. M. and Deleersnijder, E.: The boundary layer of the residence time field, Ocean Dynam., 56, 139-150, 2006.

Dimou, K. N. and Adams, E. E.: A random-walk, particles tracking models for well-mixed estuaries and coastal waters, Estuarine, Coastal and Shelf Science, 37, 99-110, 1993.

Heemink, A. W.: Stochastic modeling of dispersion in shallow water, Stochastic Hydrol. Hydraul., 4, 161-174, 1990.

Hunter, J. R.: The application of Lagrangian particle-tracking technique to modelling of dispersion in the sea, in: Numerical Mod- elling: Applications to Marine Systems, edited by: Noye, J., 257-269, 1987.

Hunter, J. R., Craig P. D., and Phillips H. E.: On the use of random walk models with spatially variable diffusivity, J. Comput. Phys., 106, 366-376, 1993.

Karatzas, I. and Shreve, S. E.: Brownian motion and stochastic calculus, Springer, New York, 1998.

Kloeden, P. E. and Platen, E.: Numerical Solution of Stochastic Differential Equations, Springer, New York, 1999.

LaBolle, E. M., Fogg, G. E., and Tompson, A. F. B.: Random-walk simulation of transport in heterogeneous porous media: Local mass-conservation problem and implementation methods, Water Resour. Res., 32(3), 583-593, 1996.

Konikow, L. F. and Bredehoeft, J. D.: Computer model of twodimensional solute transport and dispersion in ground water, U.S. Geological survey water-resources investigations book 7, Chapter C2, 1978.

LaBolle, E. M., Quastel, J., Fogg, G. E., and Granver, J.: Diffusion processes in composite porous media and their numerical integration by random walks: Generalized stochastic differential equations with discontinuous coefficients, Water Resour. Res., 36(3), 651-662, 2000.

Oksendal, B.: Stochastic differential equations, Springer, NewYork, 1985.

Proehl, J. A., Lynch, D. E., McGillicuddy Jr., D. J., and Ledwell, J. R.: Modelling turbulent dispersion of the North Flank of Georges Bank using Lagrangian Methods, Cont. Shelf Res., 25, 875-900, 2005.

Ross, O. N. and Sharples, J.: Recipe for 1-D Lagrangian particle tracking models in space-varying diffusivity, Limnol. Oceanogr: Methods 2, 289-302, 2004.

Sawford, B. L: Recent developments in the Lagrangian stochastic theory of turbulent dispersion, Bound.-Lay. Meteorol., 62, 197215, 1993.

Spivakovskaya, D., Heemink, A. W., Milstein, G. N., and Schoenmakers, J. G. M.: Simulation of the transport of particles in coastal waters using forward and reverse time diffusion, Adv. Water Resour., 28, 927-938, 2005.

Spivakovskaya, D., Deleersnijder, E., and Heemink, A. W.: Lagrangian modelling of multi-dimensional advection-diffusion with space-varying diffusivities: theory and idealized test cases, Ocean Dynam., 57, 189-203, 2007.

Stijnen, J. W., Heemink A. W., and Lin, H. X.: An efficient 3D particle model for use in stratified flow, Int. J. Numer. Meth. Fluids 51, 331-350, 2006.

Takeoka, H.: Fundamental concepts of exchange and transport time scales in a coastal sea, Cont. Shelf Res., 3, 311-326, 1984.

Thomson, D. J.: Criteria for the selection of stochastic models of particles trajectories in turbulent flow, J. Fluid Mech., 180, 529556, 1987.

Thomson, D. J., Physick, W. L., and Maryon, R. H.: Treatment of Interfaces in Random Walk Dispersion Models, J. Appl. Meteorol., 36, 1284-1295, 1997.

Visser, A. W.: Using random walk models to simulate the vertical distribution of particles in a turbulent water column, Mar. Ecol. Prog. Ser., 158, 275-281, 1997.

Yeh, G. T.: A Lagrangian-Eulerian method with zoomable hidden fine-mesh approach to solving advection-dispersion equations, Water Resour. Res., 26(6), 1133-1144, 1990. 
Zhang, R., Huang, K., and van Geruchten, M. T.: An efficient Eulerian-Lagrangian method for solving solute transport problems in steady and transient flow fields, Water Resour. Res., 28(12), 4131-4138, 1993.

Zheng, C. and Bennett, G. D.: Applied contaminant transport modeling, Wiley, 2002.

Zheng, C. and Wang, P. P.: MT3DMS: A Modular ThreeDimensional Multispecies Transport Model for Simulation of Advection, Dispersion and Chemical Reactions of Contaminants in Groundwater Systems; Documentation and User's Guide, Contract Report SERDP-99-1, U.S. Army Engineer Research and Development Center, Vicksburg, MS, 1999.
Zimmerman, J. F. T.: Mixing and flushing of tidal embrayments in the Western Dutch Wadden Sea, Part I: distribution of salinity and calculation of mixing time scales, Neth. J. Sea Res., 10, 149191, 1976.

Zimmerman, J. F. T.: Estuarine residence time, in: Hydrodynamics of estuaries, edited by: Kjerve, B., 1, 75-84, CRC Press, Boca Raton, FL, 1988

Zimmermann, S., Koumoutsakos, P., and Kinzelbach, W.: Simulation of pollutant transport using a particle method, J. Comput. Phys., 173(1), 322-347, 2001. 